\title{
Expression and regulation of bone morphogenetic protein 4 in hepatocellular carcinoma
}

\author{
Xiaotong Wang ${ }^{1,2,4}$, Qingguo Wang ${ }^{2}$, Weisi Kong ${ }^{1}$, Gerald Y. Minuk ${ }^{3}$, Frank J. Burczynski ${ }^{1}$, Emmanuel Ho ${ }^{1}$ and Yuewen Gong ${ }^{1,3 *}$ \\ ${ }^{1}$ Faculty of Pharmacy, University of Manitoba, Winnipeg, Manitoba, Canada \\ ${ }^{2}$ Department of TCM Basic Clinical Research, Beijing University of Chinese Medicine, Beijing, China \\ ${ }^{3}$ Section of Hepatology, Department of Internal Medicine, Faculty of Medicine, University of Manitoba, Winnipeg, Manitoba, Canada \\ ${ }^{4}$ Department of Rheumatology in Traditional Chinese Medicine, China-Japan Friendship Hospital, Beijing, China
}

\begin{abstract}
Bone morphogenetic protein 4 (BMP4), a member of the transforming growth factor-beta superfamily, has been implicated in the pathogenesis of hepatocellular carcinoma (HCC). Human HCC tissues, adjacent non-tumor tissues and three HCC cell lines (Huh-7, HepG2 and PLC/PRF/5) were employed for evaluation of BMP4 and BMP type I (BMPR1A and BMPR1B) and type II (BMPR2) receptor mRNA expression. In addition Huh-7 cells were further studied to document the effects of toxin-induced injury on BMP4 mRNA and protein expression. The effects of exogenous BMP4 on Huh-7 Smad 1 and ERK $1 / 2$ signaling pathways, proliferation and differentiation were determined. The results of the study revealed that BMP4 mRNA expression is upregulated in the majority of HCC and malignant hepatocyte cell lines while BMP type I and II receptor mRNA expression is more variable. Moreover, D-galactosamine induced injury of Huh-7 cells results in significant upregulation of BMP4 mRNA and protein expression. Finally, exogenous BMP4 induces phosphorylation of Smad 1 and ERK $1 / 2$ without altering cell proliferation or differentiation. These findings suggest that BMP4 is involved in the pathogenesis of HCC but by mechanisms other than regulating malignant hepatocyte proliferation or differentiation.
\end{abstract}

\section{Introduction}

Hepatocellular carcinoma (HCC) is the fifth most common cancer and third most common cause of cancer-related death in the world today. Unfortunately, potentially curative treatments such as liver transplantation and surgical resection can only be offered to a minority of HCC patients. However, more effective therapeutic interventions will only be identified when our understanding of HCC carcinogenesis is more complete.

Bone morphogenetic protein 4 (BMP4) is initially identified in the 1960s and belongs a member of the transforming growth factor beta (TGF-beta) superfamily. At present, 15 BMPs and 4 related growth differentiation factors (GDFs) have been identified that shares a similar structure $[1,2]$. Biologically active BMPs are 30-38 $\mathrm{kDa}$ homodimers that are synthesized as prepropeptides with 400-525 amino acids in cells [3]. The disulfide-linked mature dimer of BMPs will associate with membrane receptors that have been identified as type I receptors - BMPR1A (or activin receptor-like kinase 3 - ALK3) and BMPR1B (or ALK6), and type II receptor - BMPRII [1]. Binding of a BMP dimer to its type II receptor recruits the type I receptor. A heterotetramer is then formed with two receptors of each type. Proximity of the receptors allows the type II receptor to phosphorylate the type I receptor at the GS domain. This is followed by phosphorylation of Smads and/ or mitogen-activated protein kinases (MAPKs). Phosphorylated Smads and MAPKs subsequently induce intracellular events that lead to regulation of target gene transcription [3]. Biological activities of BMP4 involves in cell proliferation, differentiation, apoptosis and general organogenesis. Recently studies reveal that BMP4 may induce migration and invasion of colon [4,5], ovarian [6] and pancreatic cancer cells [7]. In addition, inhibition of BMP4 in hepatocellular carcinoma and melanoma cells resulted in reduced migration and invasion $[8,9]$.
Moreover, BMP4 mRNA and protein were found increased in HCC tumor tissues compared to adjacent cirrhotic tissues and may be related to poor prognosis in patients with HCC [10].

However, what are functions and role of BMP4 in HCC still remain unclear. Therefore, in the current study, we documented BMP4 mRNA expression in HCC tissues and cell lines. In addition we demonstrated regulation of BMP4 expression in HCC cell line (Huh-7 cells) and effects of BMP4 on intracellular signaling, proliferation and differentiation of Huh-7 cells.

\section{Materials and methods}

\section{Materials}

HCC cell lines (Huh-7, HepG2 and PLC/PRF/5 cells) were purchased from ATCC. All reagents for cell culture (Dulbecco's modified Eagle medium (DMEM), fetal bovine serum (FBS), trypsin-EDTA) and TRIzol Reagent were purchased from Invitrogen (Burlington, ON). Polymerase chain reaction (PCR) primers were designed by the Oligo 7 computer software and synthesized by Invitrogen. Donkey anti-rabbit IgG, rabbit anti-mouse IgG and the enhanced chemiluminescence (ECL) Western blotting kit were purchased from the GE Health Science

Correspondence to: Yuewen Gong, Ph.D., B.M., Faculty of Pharmacy, University of Manitoba, 750 McDermot Avenue, Winnipeg, Manitoba, Canada, R3E 0T5, Tel: 1-204-474-9359; E-mail: Yuewen.Gong@ad.umanitoba.ca

Key words: bone morphogenetic protein 4, hepatocellular carcinoma, D-galactosamine, gene regulation

Received: January 12, 2015; Accepted: January 26, 2015; Published: January 29, 2015 
(Baie d'Urfe, Quebec). The iScript ${ }^{\mathrm{TM}} \mathrm{cDNA}$ Synthesis Kit and $\mathrm{iQ}^{\mathrm{TM}}$ $\mathrm{SYBR}^{\circ}$ Green Supermix were purchased from Bio-Rad Laboratories (Canada) Ltd. (Mississauga, Ontario). D-galactosamine (D-gal) and other chemicals were purchased from Sigma-Aldrich Co. (Oakville $\mathrm{ON})$.

\section{Human hepatocellular carcinoma tissue samples}

Total RNA from human hepatocellular carcinoma HCC and adjacent non-tumor tissues were obtained from our previous study [11]. That study was approved by the University of Manitoba Conjoint Ethics Committee for Human and Animal Experimentation.

\section{Human hepatocellular carcinoma cells and cell culture}

All HCC cells were cultured in DMEM supplemented with $110 \mathrm{mg} / \mathrm{L}$ sodium pyruvate, $10 \%$ fetal bovine serum (FBS), $100 \mathrm{U} /$ $\mathrm{ml}$ penicillin and $100 \mu \mathrm{g} / \mathrm{ml}$ streptomycin at $37^{\circ} \mathrm{C}$ in a humidified atmosphere of $5 \% \mathrm{CO}_{2}$ and $95 \%$ air. Huh-7 cells were sub-cultured in $10 \mathrm{~cm}$ culture dishes for experiments. One day prior to treatment, culture medium was removed and cells were incubated with the medium described above supplemented with different concentrations of $\mathrm{D}$-gal for different time intervals as indicated.

\section{Extraction of RNA and performance of real time reverse transcriptase polymerase chain reaction (RT-PCR)}

Total RNA from tissues and/or cells was isolated by TRIzol reagent according to the manufacturer's manual. The first strand cDNA was synthesized by the iScript ${ }^{\mathrm{TM}} \mathrm{cDNA}$ Synthesis Kit as described previously [12]. PCR was performed using the $\mathrm{iQ}^{\mathrm{TM}} \mathrm{SYBR}^{\circ}$ Green Supermix and the oligonucleotides were synthesized by Invitrogen. The specific primers for the study are listed in Table 1 and were designed with the respective sequences from GenBank by Oligo 7 software. PCR amplification was carried out by applying 30 cycles comprising: denaturation at $94^{\circ} \mathrm{C}$ for 1 minute, different annealing temperatures for 30 seconds, elongation at $72^{\circ} \mathrm{C}$ for 2 minutes, followed by a final elongation at $72^{\circ} \mathrm{C}$ for 8 minutes using a MiniOpticon ${ }^{\mathrm{TM}}$ Real-Time PCR Detection System (Bio-Rad Laboratories (Canada) Ltd. Mississauga, Ontario). PCR products were also analyzed by electrophoresis on a 1.2\% agarose gel.

\section{Extraction of cellular protein and Western blot analyses}

Cellular protein was isolated by a cellular protein extraction solution (1 $\mathrm{X}=50 \mathrm{mM}$ Tris pH8.0, $0.5 \mathrm{mM}$ EDTA, $150 \mathrm{mM} \mathrm{NaCl}, 0.5 \% \mathrm{NP}$ 40 and $1 \mathrm{X}$ protease inhibitor cocktail (Sigma-Aldrich, Oakville ON). The BCA protein assay kit measured protein concentrations. Twenty micrograms of cellular protein from different samples were then mixed with $4 \mathrm{X}$ gel loading buffer, separated on $12 \%$ sodium dodecyl sulfatepolyacrylamide (SDS-polyacrylamide) gel under reducing conditions, and transferred onto Nitroplus-2000 membranes (Micron Separations

Table 1: Primers and conditions of polymerase chain reaction.

\begin{tabular}{|l|l|l|c|c|}
\hline Genes & \multicolumn{1}{|c|}{ Primers } & Tm $\left({ }^{\circ} \mathbf{C}\right)$ & Size (bp) \\
\hline BMP4 & sense & 5'-CCGGGAAAAGCAACCCAAC-3' & 59.6 & 286 \\
\hline & anti-sense & 5'-GCCCACGTCACTGAAGTCCA-3' & & \\
\hline Albumin & sense & 5'-CTATGCCAAAGTGTTCGAT-3' & 53.1 & 299 \\
\hline & anti-sense & 5'-CTGGCGTTTTCTCATGCAA-3' & & \\
\hline Cytokeratin 19 & sense & 5'-ACAGCCACTACTACACGACCA-3' & 58.5 & 169 \\
\hline & anti-sense & 5'-ATGTCGGCCTCCACGCTCA-3' & & \\
\hline GAPDH & sense & 5'-CAAAGTGGACATTGTTGCCAT-3' & 56.2 & 204 \\
\hline & anti-sense & 5'-ATACTAGCACCAGCATCACC-3' & & \\
\hline$\beta$-actin & sense & 5'-GCACCACACCTTCTACAATG-3' & 60 & 838 \\
\hline & anti-sense & 5'-TGCTTGCTGATCCACATCTGS-3' & & \\
\hline
\end{tabular}

Inc. Westborough, MA). Nonspecific antibody binding was blocked by pre-incubation of the membranes in $1 \mathrm{X}$ Tris-buffered-saline (TBS) containing $5 \%$ skim milk for 1 hour at room temperature. Membranes were incubated overnight at $4^{\circ} \mathrm{C}$ with antibodies against respective proteins - monoclonal antibody against BMP4 at 1:500 dilution, monoclonal antibody against Smad1 at 1:500 dilution, polyclonal antibody against phosphor-Smad1 at 1:700 dilution, polyclonal antibody against ERK1/2 at 1:800 dilution, polyclonal antibody against phosphor-ERK1/2 at 1:400 dilution and monoclonal antibody against $\beta$-actin at 1:6000 dilution respectively in $1 \mathrm{X}$ TBS containing $0.1 \%$ Tween-20 and $2 \%$ skim milk. After washing, they were incubated with donkey anti-rabbit IgG or sheep anti-mouse IgG at 1:1000 dilutions for 1 hour at room temperature, respectively. Bands were visualized by employing the enhanced chemiluminescence kit according to the manufacturer's instructions.

\section{Cell proliferation assay}

Cell proliferation was measured using cell proliferation reagents WST- 1 as described previously [13]. Huh- 7 cells $\left(2 \times 10^{3}\right)$ in $200 \mu$ l culture medium were seeded into 96 well plates. After 24 hours incubation, the medium was changed with $100 \mu$ l of fresh culture medium containing different concentrations of D-gal or BMP4 or combination of D-gal and BMP4. The media as well as drugs were changed once every other day. Cell proliferation was documented after 1, 3, 6, 9 days of treatments. At the end of treatment, $10 \mu$ of WST- 1 reagent were added into wells and incubated for 3 hours. The absorbency of the treated samples against a blank control was measured using a Synergy HT microplate reader (BioTek Instruments) with $420 \mathrm{~nm}$ as detection wavelength/650nm as reference wavelength for WST-1 assay. Each treated group contained 8 wells and each experiment was repeated on three occasions.

\section{Statistical analyses}

To analyze differences between control and treatment groups, we performed the ANOVA and Fisher's PLSD test as Post hoc test using StatView (version 5.0) software (SAS Institute Inc. Cary, NC). Differences with $\mathrm{p}$ value below 0.05 were considered statistically significant.

\section{Results}

Although BMP4 expression in HCC has been reported, the interactive proteins with BMP4 such as its receptors have not been described. Our previous study with human HCC tumors in other project allowed us to investigate the expression of BMP4, BMPR1A, BMPR1B and BMPR2 mRNA at same time. Expression pattern of BMP4 and its receptors in five HCC and adjacent non-tumor tissues from 12 HCC samples were shown in Figure 1. BMP4, BMPR1A, BMPR1B and BMPRII were all expressed in HCC samples. Compared to adjacent non-tumor tissues, BMP4 mRNA expression was significantly increased in HCC tumor tissues as well as BMPR1A. The mRNA level of BMPRII was also elevated but there is no statistical significance. Moreover, there was no change of BMPR1B mRNA between tumor and adjacent non-tumor tissues.

Although there is increased expression of BMP4 in HCC tumor tissues compared to that of adjacent non-tumor tissues, the role of BMP4 in HCC has yet to be investigated. Because malignant hepatocytes are often exposed to ischemia, physical ablation with heat or cold and/ or chemotherapy, we designed additional experiments wherein the effects of toxin-induced injury on BMP4 expression were documented in Huh-7 cells. As shown in Figure 2, following exposure to $4.0 \mathrm{mM}$ 


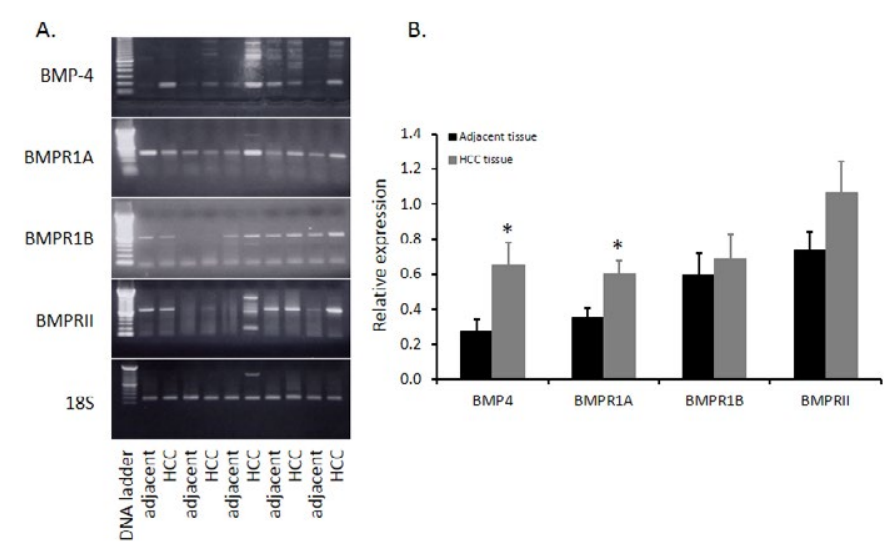

Figure 1. Comparison of BMP4 and BMPRs mRNA in HCC tissues and adjacent nontumor tissues. Twelve human HCC were collected in our previous study and total RNA from HCC tissues and adjacent non-tumor tissues were extracted and BMP4 mRNA was evaluated by RT-PCR. Five paired samples were shown in panel A and abundance of BMP4 in twelve paired samples was shown in panel B. Data were presented as mean $\pm \mathrm{SE}$ and * indicates significant difference between HCC tissues and adjacent non-tumor tissues.

A.
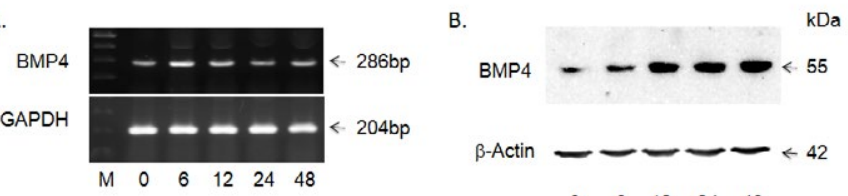

$\beta$-Actin $-\longrightarrow-\longrightarrow$

$\begin{array}{lllll}0 & 6 & 12 & 24 & 48\end{array}$

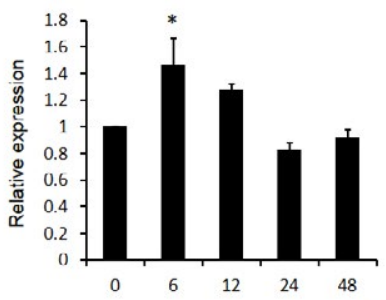

Time of treatment (hours)

Figure 2. D-gal induced bone morphogenetic protein 4 (BMP4) expression in Huh-7 cells over time. Panel A shows significant increase in BMP4 mRNA and panel B shows the abundance of BMP4 protein after D-gal treatment. The top panels display representative gels of BMP4 mRNA and protein. The bottom panels display histogram of these bands. Data are presented as mean \pm SEM from four different experiments. * indicates statistically significant difference between D-gal treated and untreated cells.

of the hepatotoxin - D-galactosamine, the increase in BMP4 mRNA expression peaked at 6 hours, prior to returning to baseline levels at 24 hours. BMP4 protein expression was significantly increased at 12 hours and continued to increase until the end of the experiment at 48 hours. This effect on BMP4 mRNA and protein expression was also concentration dependent in that no significant effects were observed following exposure to $0.4 \mathrm{mM}$ D-galactosamine but significant increases were obtained with $4.0 \mathrm{mM}$ and the greatest effect with 8.0 $\mathrm{mM}$ of toxin (Figure 3).

To determine whether increases in BMP4 expression are likely to be associated with changes in cell signaling, we documented the effects of exogenous BMP4 on two key BMP signaling pathways; Smad 1 and ERK 1/2 in Huh-7 cells. As shown in Figure 4, BMP4 treatment resulted in significant increases in Smad 1 phosphorylation at 0.5 and 1 hour while increases in ERK $1 / 2$ phosphorylation were present at all three time points: $0.5,1$ and 2 hours.
BMP4 administration has been reported to alter malignant cell proliferative activity. It has also been reported to enhance cell differentiation. In our study, D-galactosamine significantly inhibited Huh-7 cell proliferation in a dose dependent mansion (Figure 5A). However, various concentrations of BMP4 alone had no effect on Huh-7 proliferation (Figure 5B) and combination of BMP4 or BMP4 antibody did not alter the inhibitory effect of $\mathrm{D}$-galactosamine on Huh-7 cell proliferation (Figure 5C). There was no effect of D-gal on cell differentiation as indicated by the expression of albumin (marker of hepatocyte differentiation) and CK-19 (marker of cholangiocyte differentiation) (data not shown).

\section{Discussion}

The results of this study confirm previous reports that BMP4 expression is increased or abundant in a majority of HCC tissues and malignant hepatocyte cell lines. Also in keeping with previous reports was the variability in BMP receptor expression, with BMP Type I and II receptor expression being increased, decreased or unchanged in various HCC tissues and malignant hepatocyte cell lines. The results
A.
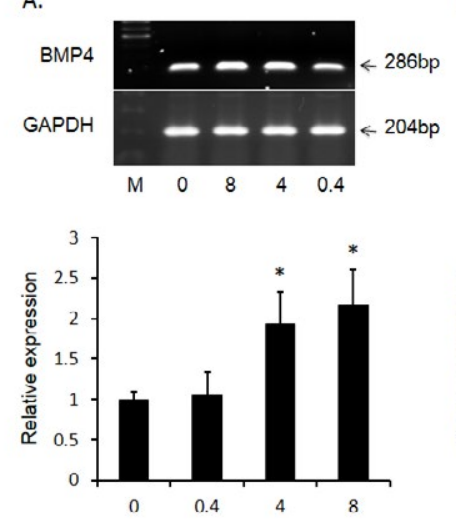

Concentration of D-Gal (mM)
B.

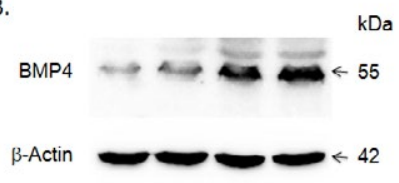

$\begin{array}{llll}0 & 0.4 & 4 & 8\end{array}$

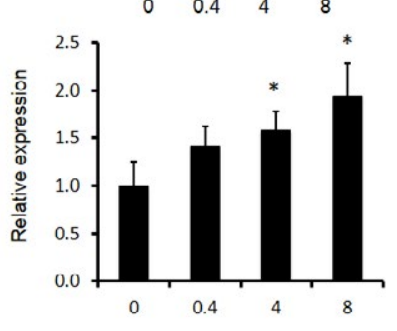

Figure 3. D-gal induced bone morphogenetic protein 4 (BMP4) expression in Huh-7 cells as a function of D-gal concentration. Panel A displays significant increase in BMP4 mRNA and panel B shows BMP4 protein levels after treatment with different concentrations. The top panels display representative gels of BMP4 mRNA and protein. The bottom panels display histogram of these bands. Data are presented as mean \pm SEM from four different experiments and $*$ indicates statistically significant difference between the treated cells to the untreated cells.

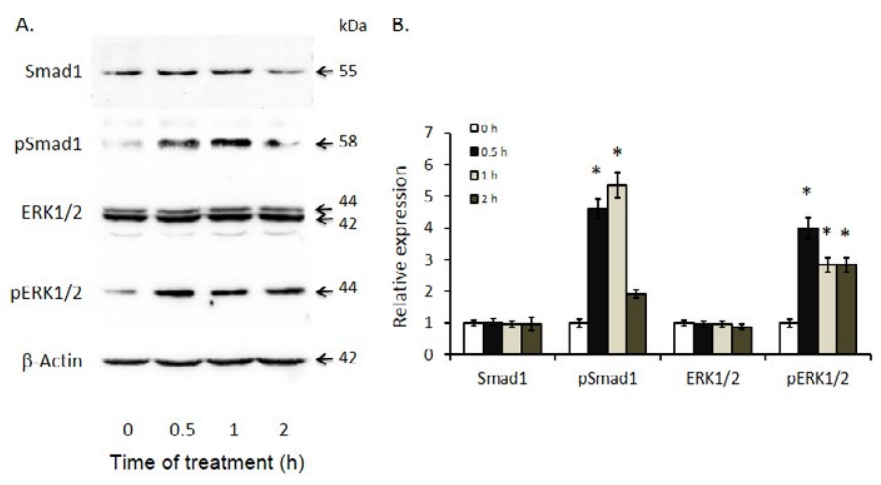

Figure 4. Induced Smad 1 and ERK 1/2 phosphorylation after bone morphogenetic protein 4 (BMP4) treatment of Huh-7 cells. Smad1, phosphor-Smad1, ERK1/2 and phosphorERK1/2 expression were documented by Western blot analyses with antibodies against the respective proteins. The left panel displays representative Western blot gels and the right panel shows histograms of band densities. Data are presented as mean \pm SEM from four different experiments. $*$ indicates statistically significant differences between the different time intervals of treatment to baseline (0 hours). 
A.

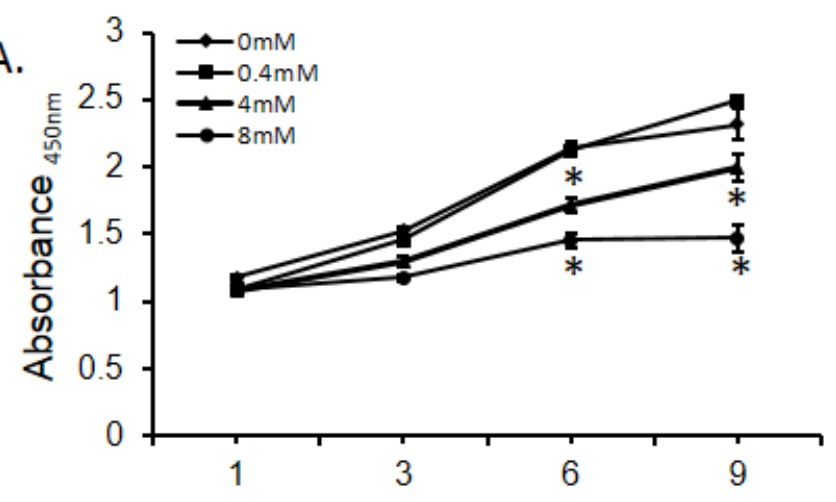

B.

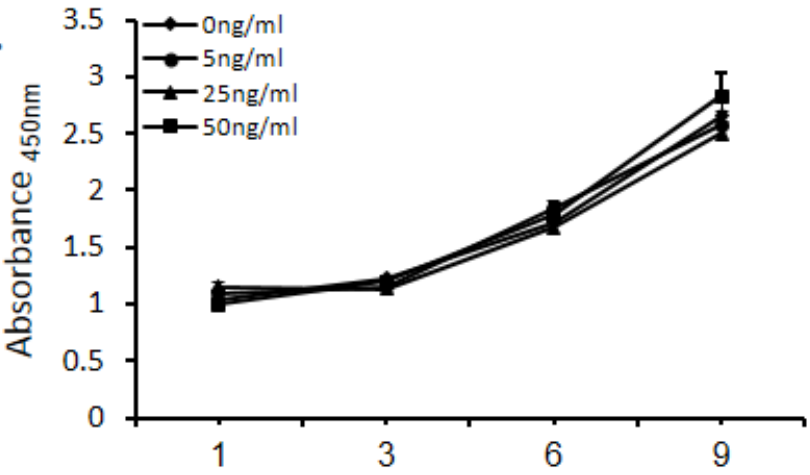

C.

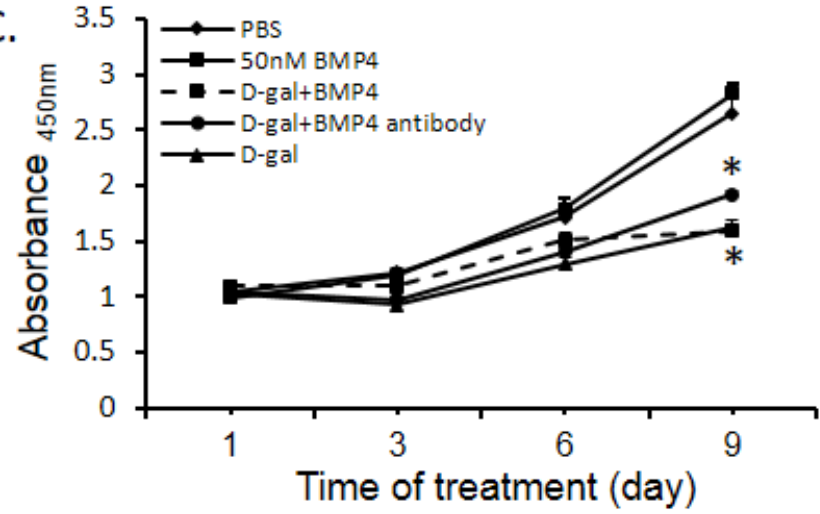

Figure 5. Regulation of Huh-7 cell proliferation by bone morphogenetic protein 4 (BMP4). Panel A shows the effects of D-gal on Huh-7 cell proliferation. Panel B displays the BMP4 regulation of Huh-7 cell proliferation. Panel C shows the effects of D-gal (4 mM), BMP4 $(50 \mathrm{ng} / \mathrm{ml})$, BMP4 antibody $(400 \mathrm{ng} / \mathrm{ml})$ and the combination of D-gal and BMP4 on Huh7 cell proliferation. Data are presented as mean \pm SEM from six different wells and two different experiments. * indicates statistically significant difference between D-gal treated and un-treated cells.

also extend our understanding of BMP4 regulation by demonstrating that in addition to hypoxia, BMP4 expression is upregulated by toxininduced injury. Finally, despite significant increases in BMP signaling pathways, we were unable to confirm previous reports of BMP4 altering malignant cell proliferation and/or differentiation.

There is a paucity of reports describing BMP4 and BMP receptor expression in human HCC. In the first of only three such reports published to date, Maegdefrau et al. described BMP4 expression in 39 paired tissue specimens from HCC patients and found that in $62 \%$, expression was upregulated [8]. Shortly thereafter, Guo et al. described increased BMP4 protein expression by immunohistochemistry in 120/156 (77\%) HCC tissues compared to 19/156 (12\%) adjacent nontumor tissues [10]. Most recently, Chiu et al. reported increased BMP4
mRNA expression by real-time PCR in $60 \%$ of 71 paired HCC tissues [14]. In the same study, Chiu found that in 30/71 (43\%) specimens BMPR1A mRNA expression was upregulated, while BMPR1B expression was down regulated in all 71 specimens (BMPR2 expression was not reported in their study). Although the number of paired HCC specimens was much smaller $(\mathrm{N}=15)$ in the present study, our results are in keeping with the above findings in that BMP4 mRNA expression was increased in the majority of HCC specimens while BMP type I and II receptor expression was quite variable. Given what is now known about BMP4 regulation, it will be important to document the conditions associated with sample collection (e.g. intra-operative ischemia, recent chemotherapy, ablations, etc.) prior to determining BMP4 and/or BMP4 receptor expression in future studies.

In the only study reporting BMP4 expression in malignant hepatocyte cell lines, Maegdefrau et al. described a 4-5 fold upregulation of BMP4 mRNA in Hep3B, HepG2 and PLC cells relative to primary human hepatocytes [8]. Because we did not culture primary human hepatocytes in the present study, no such comparisons could be made. However, we did document abundant BMP4 expression in Huh-7 and to a lesser extent, PLC/PRF/5 cells. Why expression was so limited in HepG2 cells is unclear. Again, given what is now known about BMP4 expression, differences in culture conditions, number of cell passages, cell viability etc. must be considered as a possible explanation for these discordant findings.

$\mathrm{D}$-galactosamine is a potent hepatotoxin that induces hepatocyte injury by causing an accumulation of UDP-galactosamine derivatives in the liver [15]. These in turn lead to a depletion of hepatic UTP and cessation of the biosynthesis of macromolecules such as RNA, protein, glycoproteins, glycogen etc. Thus, many mechanisms of cell injury are represented by this toxin. Together with previous data indicating that ischemia results in an upregulation of BMP4 expression in malignant hepatocytes, the results of our D-galactosamine experiments suggest that regardless of the nature of the insult, BMP4 expression is upregulated. It is tempting to speculate that this finding could explain the high incidence of tumor recurrences following ischemic injury (as occurs with transarterial chemoembolization) and/or systemic chemotherapy by invoking BMP4 activation of chemoresistant cancer stem/progenitor cells or angiogenesis within the tumor tissue.

Intracellular signaling molecules play an important role in cytokine regulation of cell proliferation and differentiation. There are two intracellular signaling pathways that mediate BMP4 signal transduction - Smad 1 and ERK1/2. BMP4-activated Smad 1 translocates into the nucleus and recruits the transcription of co-activators cAMP responsive element-binding protein (CREB) and p300, which subsequently induce the expression of hepatic differentiation genes in hepatic stem cells [16]. The results of the present study demonstrate that BMP4 induces significant phosphorylation of Smad 1 in Huh-7 cells. The other intracellular signaling pathway of BMP4 is the mitogen activated protein kinase (MAPK) pathway. This pathway includes ERK1/2, which mediates cell proliferation and differentiation [17]. FGFactivated ERK1/2 signaling is responsible for initiation of hepatic gene expression related differentiation in embryonic endoderm cells [18]. In the present study we found that in addition to Smad 1 activation, D-galactosamine induced injury of Huh-7 cells also activates ERK1/2 signaling.

Despite the induction of these signaling pathways we did not observe an alteration in either hepatocyte proliferation or differentiation following treatment with exogenous BMP4. The 
absence of an effect on proliferation is in keeping with results reported by Maegdefrau et al. [8] but contrary to those of Chiu et al. [14]. However, it should be noted that in the latter study, proliferative activity was described as increased but the increase was limited and statistical analyses were applied. Regarding cell differentiation, in the present study BMP4 did not alter albumin or CA19 mRNA expression, reflecting hepatocyte and cholangiocyte differentiation respectively. Perhaps most relevant to this finding is the use of Huh-7 cells which are considered well differentiated and therefore, less likely to undergo further differentiation as opposed to de-differentiation.

In conclusion, the results of this study support the hypothesis that BMP4 is involved in the pathogenesis of HCC. However, the precise role BMP4 plays remains unclear. Given that despite a significant increase in BMP4 expression and signaling, malignant hepatocyte proliferation and differentiation remain unaltered, future studies should focus on the effects of BMP4 on cancer stem/progenitor cells or non-hepatocellular tumor features such as angiogenesis.

\section{Acknowledgement}

This research was supported by the grant (RGPIN/355304-2010) from the Natural Science \& Engineering Research Council to Dr. Yuewen Gong and the Graduate Scholarship from the China Scholarship Council to Ms. Xiaotong Wang.

\section{References}

1. Yamashita H, Ten Dijke P, Heldin CH, Miyazono K (1996) Bone morphogenetic protein receptors. Bone 19: 569-574. [Crossref]

2. Aldinger G, Herr G, Küsswetter W, Reis HJ, Thielemann FW, et al. (1991) Bone morphogenetic protein: a review. Int Orthop 15: 169-177. [Crossref]

3. Wozney JM (1998) The bone morphogenetic protein family: multifunctional cellular regulators in the embryo and adult. Eur J Oral Sci 106: 160-166. [Crossref]

4. Deng H, Makizumi R, Ravikumar TS, Dong H, Yang W, et al. (2007) Bone morphogenetic protein- 4 is overexpressed in colonic adenocarcinomas and promotes migration and invasion of HCT116 cells. Exp Cell Res 313: 1033-1044. [Crossref]

5. Deng H, Ravikumar TS, Yang WL (2009) Overexpression of bone morphogenetic protein 4 enhances the invasiveness of Smad4-deficient human colorectal cancer cells. Cancer Lett 281: 220-231. [Crossref]
6. Thériault BL, Shepherd TG, Mujoomdar ML, Nachtigal MW (2007) BMP4 induces EMT and Rho GTPase activation in human ovarian cancer cells. Carcinogenesis 28: 1153-1162. [Crossref]

7. Gordon KJ, Kirkbride KC, How T, Blobe GC (2009) Bone morphogenetic protein induce pancreatic cancer cell invasiveness through a Smad1-dependent mechanism that involves matrix metalloproteinase-2. Carcinogenesis 30: 238-248. [Crossref]

8. Maegdefrau U, Amann T, Winklmeier A, Braig S, Schubert T, et al. (2009) Bone morphogenetic protein 4 is induced in hepatocellular carcinoma by hypoxia and promotes tumour progression. J Pathol 218: 520-529. [Crossref]

9. Rothhammer T, Poser I, Soncin F, Bataille F, Moser M, et al. (2005) Bone morphogenic proteins are overexpressed in malignant melanoma and promote cell invasion and migration. Cancer Res 65: 448-456. [Crossref]

10. Guo X, Xiong L, Zou L, Zhao J (2012) Upregulation of bone morphogenetic protein 4 is associated with poor prognosis in patients with hepatocellular carcinoma. Pathol Oncol Res 18: 635-640. [Crossref]

11. Minuk GY, Zhang M, Gong Y, Minuk L, Dienes H, et al. (2007) Decreased hepatocyte membrane potential differences and GABAA-beta3 expression in human hepatocellular carcinoma. Hepatology 45: 735-745. [Crossref]

12. Shen H, Huang G, Hadi M, Choy P, Zhang M, et al. (2003) Transforming growth factor-beta1 downregulation of Smad1 gene expression in rat hepatic stellate cells. $\mathrm{Am}$ J Physiol Gastrointest Liver Physiol 285: G539-546. [Crossref]

13. Shen H, Huang GJ, Gong YW (2003) Effect of transforming growth factor beta and bone morphogenetic proteins on rat hepatic stellate cell proliferation and transdifferentiation. World J Gastroenterol 9: 784-787. [Crossref]

14. Chiu CY, Kuo KK, Kuo TL, Lee KT, Cheng KH (2012) The activation of MEK ERK signaling pathway by bone morphogenetic protein 4 to increase hepatocellular carcinoma cell proliferation and migration. Mol Cancer Res 10: 415-427. [Crossref]

15. Keppler D, Lesch R, Reutter W, Decker K (1968) Experimental hepatitis induced by D-galactosamine. Exp Mol Pathol 9: 279-290. [Crossref]

16. Suzuki A, Raya A, Kawakami Y, Morita M, Matsui T, et al. (2006) Nanog binds to Smad1 and blocks bone morphogenetic protein-induced differentiation of embryonic stem cells. Proc Natl Acad Sci U S A 103: 10294-10299. [Crossref]

17. Kim J, Adam RM, Freeman MR (2002) Activation of the Erk mitogen-activated protein kinase pathway stimulates neuroendocrine differentiation in $\mathrm{LNCaP}$ cells independently of cell cycle withdrawal and STAT3 phosphorylation. Cancer research 62: 1549-1454. [Crossref]

18. Calmont A, Wandzioch E, Tremblay KD, Minowada G, Kaestner KH, et al. (2006) An FGF response pathway that mediates hepatic gene induction in embryonic endoderm cells. Dev Cell 11: 339-348. [Crossref]

Copyright: (C) 2015 Xiaotong Wang. This is an open-access article distributed under the terms of the Creative Commons Attribution License, which permits unrestricted use, distribution, and reproduction in any medium, provided the original author and source are credited. 